\title{
Comparison of reliability tests by characterization of degradation in photovoltaic modules
}

\begin{abstract}
Standard reliability tests have been developed for photovoltaic (PV) modules to assess the reliability of their performance in a short period of time. These tests generate different modes of degradation by combination of environmental stresses. There is a need to differentiate between these standard tests on the basis of severity. This helps to understand the individual and combined degradation effect occurred due to temperature and humidity stress, which is the focus of the present study. In this work, standard tests viz. humidity freeze (HF), thermal cycling (TC) and damp heat test (DH) have been performed on different batches of multi-crystalline silicon PV modules. One batch of modules was subjected to combined tests wherein these three tests were performed in succession and another batch was subjected to the individual tests. Spatial characterization techniques i.e. dark lock-inthermography (DLIT) and electroluminescence (EL) imaging were used in tandem with illuminated current voltage (I-V) analysis for fast and reliable detection and quantification of degradation caused by these tests. The application of these techniques was found to be helpful in identification of different modes of degradation after the standard tests. The study has been instrumental in understanding the severity of individual tests and their sequences along with identification of the common modes of degradation by exploiting the available characterization techniques.
\end{abstract}

Keywords: photovoltaic, multicrystalline silicon modules, reliability, degradation, defects, characterization
Volume I Issue I - 2017

\author{
Sagarika Kumar, Subinoy Roy, Rajesh Gupta \\ Department of Energy Science \& Engineering, Indian Institute of \\ Technology Bombay, India
}

\begin{abstract}
Correspondence: Rajesh Gupta, Department of Energy Science \& Engineering, Indian Institute of Technology Bombay, India, Tel +9I-22-2576-7837, Fax: +9I-22-2576-4890,
\end{abstract} Email rajeshgupta@iitb.ac.in

Received: April 29, 2017 | Published: September 20, 2017
Abbreviations: PV: photovoltaic, EL: electroluminescence, DLIT: dark lock-in-thermography, $\mathrm{P}_{\max }$ : maximum output power, $\mathrm{I}_{\mathrm{sc}}$ : short circuit current, $\mathrm{V}_{o c}$ : open circuit voltage, $\mathrm{R}_{\mathrm{s}}$ : series resistance, FF: fill factor, TC: thermal cycling, HF: humidity freeze, DH: damp heat

\section{Introduction}

The recent large scale deployment of multi crystalline silicon photovoltaic (PV) modules in the field has risen the need for in-depth studies of degradation in a fast and efficient manner. In this context, different standard test procedures as specified under IEC $61215 \mathrm{viz}$ thermal cycling (TC), humidity freeze (HF) and damp heat (DH) have been developed for crystalline silicon PV modules. ${ }^{1}$ These tests undergo different degradation mechanisms on application of various environmental stress factors in an accelerated frame and help in assessing the reliability of the modules in a short period of time. ${ }^{2,3}$ These different tests when exploited in PV modules, checks for the module reliability on different aspects such as high temperature effect, thermal ramping, and humidity ingression effect etc. These aspects essentially affect the module performance individually or in combination to one another. In order to understand the modules behavior under these stress factors, a comparative study of these standard reliability tests can be helpful. Earlier, illuminated current voltage (I-V) analysis has been used for extraction of global electrical parameters in relation to the degradation observed after these tests. ${ }^{4}$ However, I-V analysis alone cannot be used for defect analysis as it cannot map the cause of the degradation. It is essential to obtain spatially resolved information of the PV modules to understand the channel of degradation. Hereof, Electroluminescence (EL) and Dark lock-in-thermography (DLIT) in addition to non-spatial IV investigation can be used for detection and analysis of defects in PV modules. In EL imaging technique, the intensity of the EL emission is proportional to the number of minority carriers in the silicon solar cell. The number of minority carriers effects the local cell voltage which is mapped as the EL emmision in the images. ${ }^{5-7}$ In this technique, the defected regions can be spatially detected as low intensity areas or dark spots. This imaging technique is used in the production line during the fabrication process of the solar cells to identify material defects like grain boundaries and inhomogeneity caused by dislocation. Also, the post production defects induced in the solar cells such as mechanical damage to the wafer, fingers and other electrical contacts can be detected by this technique. ${ }^{8}$ In the DLIT technique the solar cell is thermally excited by passing a periodic electrical current into it. ${ }^{9}$ It can be used for detection and characterization of the defects observed in the solar cells by imaging the temperature variation over the cells due to the electrical current passed through it. This technique has not been vastly used for PV module investigation as the interpretation of the results are not straight forward as compared to solar cell applications. This technique has been used dominantely for localization of electrical defects such as shunts. ${ }^{10-12}$ They are usually process or material induced short circuits that appear bright due to the increase in flow of current accumulation, at those points after biasing in dark conditions. In this study, three standard qualification tests viz. TC, HF and DH are studied on multi-crystalline silicon PV modules followed by defect characterization by IV analysis, EL and DLIT imaging techniques. These tools have been used in tandem for the analysis of the observed degradation after the standard tests by extraction of the spatially resolved information of the defects in the modules. The test has been conducted on two module batches as shown in Figure 1. Combined tests were conducted to observe the difference in mode of degradation when conducted in succession. The tests have been compared on the basis of the observed degradation in $\mathrm{P}_{\max }$ along with correlation with images obtained after the spatial characterization of the PV modules. This study was conducted for understanding the nature of the tests and identification of their severity by analysing their effect on the PV modules. 


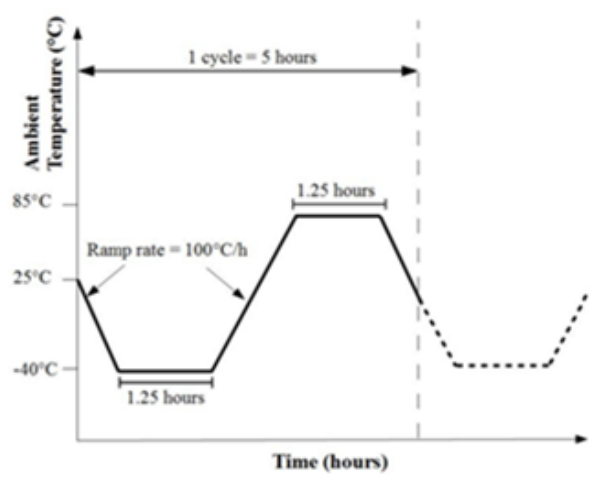

(a)

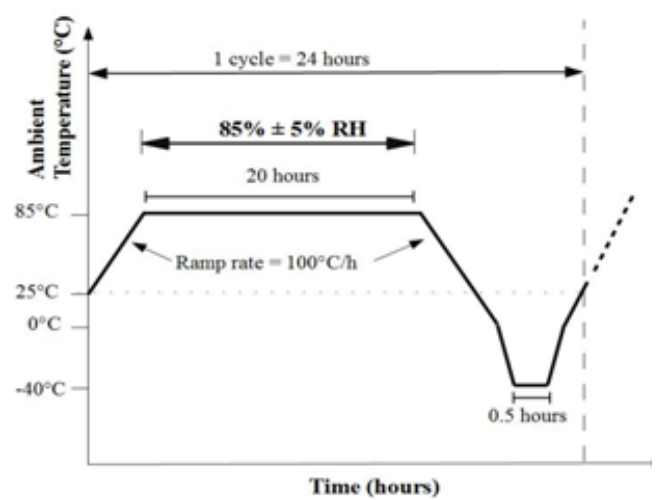

(b)

Figure I Test profile for (a) TC and (b) HF test.

\section{Materials and methods}

The study was carried out on batches of multi-crystalline silicon PV modules from the same manufacturer. The modules were chosen to have similar electrical and geometrical parameters for a proper comparative study. All the modules had an area of $0.24 \mathrm{~m}^{2}$ with 36 cell in series. The electrical parameters obtained for the study are maximum output power $\left(\mathrm{P}_{\max }\right)$, series resistance $\left(\mathrm{R}_{\mathrm{s}}\right)$, short circuit current $\left(\mathrm{I}_{\mathrm{sc}}\right)$, fill factor $(\mathrm{FF})$ and open circuit voltage $\left(\mathrm{V}_{\mathrm{oc}}\right)$. Standard tests procedures have been performed on multi-crystalline silicon PV modules, whose test conditions have been described in Figure 1. Initially, the modules have been visually inspected and pre-characterized by I-V, EL and DLIT techniques. These measurements were used as reference for the degradation study. Sample modules have been divided in two batches, batch-I was subjected to these three tests in succession in the order HF, TC and DH for similar duration of operation. In batch-II different modules were subjected to the individual tests separately. The detailed test sequence has been described in Figure 2. TC test consisting of a sequence of 50 cycles, of 5 hours each was conducted at a temperature of $+85^{\circ} \mathrm{C}$ to $-40^{\circ} \mathrm{C}$ with a ramp rate of $1.67^{\circ} \mathrm{C} /$ min, as shown in Figure 1(a). HF test consisting of a sequence of 10 cycles, of 24 hours each was carried out at a temperature of $+85^{\circ} \mathrm{C}$ to $-40^{\circ} \mathrm{C}$ at similar ramp rate as TC along with 1 hour of dwell period at $85^{\circ} \mathrm{C} / 85 \% \mathrm{RH}$, as shown in Figure $1 \mathrm{~b}$. DH test was performed at constant $85^{\circ} \mathrm{C} / 85 \% \mathrm{RH}$ for 250 hours. The tests were performed in an environmental chamber, which was dedicated to run these standard tests on PV modules. Characterizations by EL imaging and DLIT technique have been used for investigation of defects after every step of the sequence. The measurements have been taken at constant room temperature. For EL imaging, a silicon based charged couple device (CCD) camera has been used in a dark enclosure. The $1024 \times 1024$ pixel silicon CCD detector used in the camera was sensitive in the range of 300-1050 $\mathrm{nm}$. The PV modules were forward biased at short circuit current value $\left(\mathrm{I}_{\mathrm{sc}}\right)$ by a programmable power supply. For DLIT experimental measurements, thermal images of the modules have been obtained by a $320 \times 256$ pixels cooled indium antimonide (InSb) detector IR camera operating in the spectral range of 3-5 $\mu \mathrm{m}$. The modules were forward biased at $I_{\text {sc }}$ by a programmable power supply.

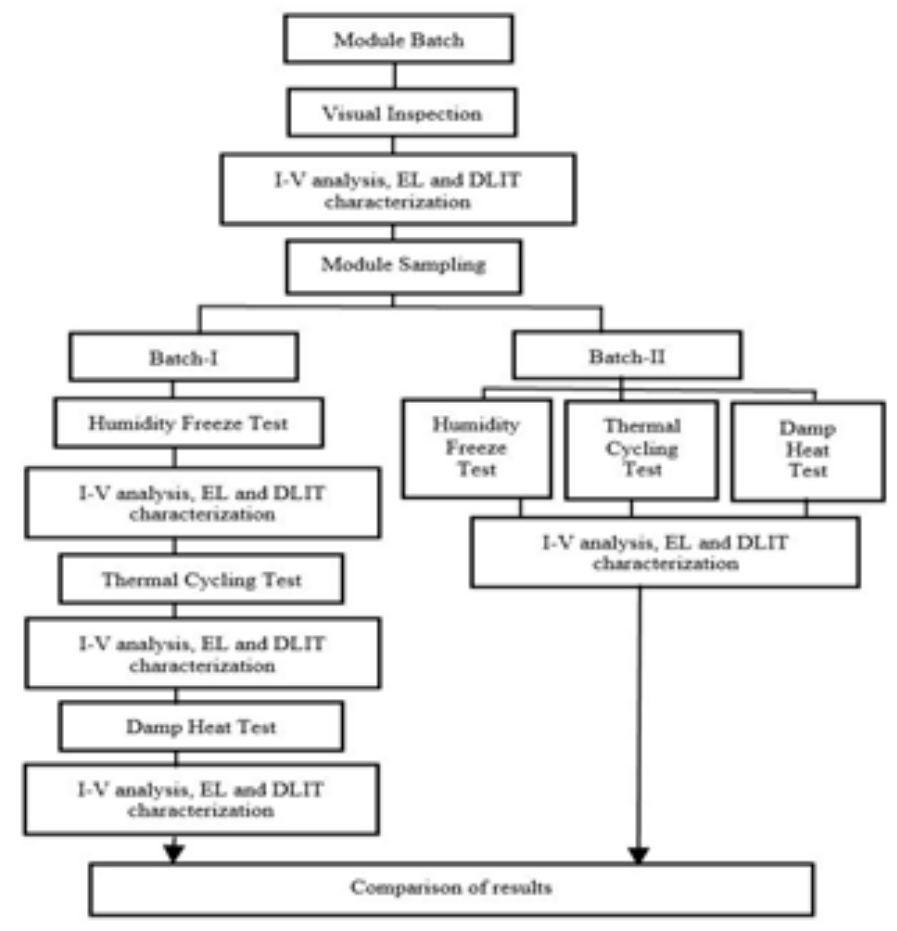

Figure 2 Experimental methodology. 


\section{Results and discussion}

In this section, the experimental results after DLIT and EL imaging are discussed for analysis of degradation observed after the standard reliability tests. $\mathrm{I}-\mathrm{V}$ analysis has been discussed to investigate the preliminary signs of degradation and has been correlated with the results obtained from the EL and DLIT images.

\section{Illuminated current voltage (I-V) analysis}

TC, HF and DH were performed for a time span of 250 hours. Visual inspection of the PV modules did not reveal any defects visible to the naked eye after the tests. The loss in the electrical parameters for sample modules after each test has been shown in Table 1. The $\mathrm{I}-\mathrm{V}$ analysis showed significant degradation in maximum output power $\left(\mathrm{P}_{\max }\right)$ for all the modules. Here, $\mathrm{P}_{\max }$ was observed to degrade in the decreasing order of TC, HF and DH for the batch-II modules where modules were subjected to the individual tests separately. The variation in $\mathrm{I}_{\mathrm{sc}}$ and $\mathrm{V}_{\mathrm{oc}}$ was observed to be non-significant $(<1 \%)$ for the duration of the test. Loss in fill factor (FF) was seen as the major contributor for the loss in output power. One to one correspondance was observed between the values of $\mathrm{FF}$ and $\mathrm{P}_{\max }$. The loss in FF loss has been attributed to the increment in series resistance $\left(\mathrm{R}_{\mathrm{s}}\right)$. The maximum degradation in $\mathrm{P}_{\max }$ was observed after TC $(\sim 7 \%)$ where the $\mathrm{R}_{\mathrm{s}}$ increased the most $(\sim 20 \%)$, on the other hand the loss in power after $\mathrm{DH}$ was observed to be about $2 \%$ with an increment in $\mathrm{R}_{\mathrm{s}}$ of almost $5 \%$. The $\mathrm{I}-\mathrm{V}$ analysis of the batch-I modules also showed similar results where $\mathrm{P}_{\max }$ was observed to degrade in the decreasing order of TC, HF and DH and the reason for loss in $\mathrm{P}_{\max }$ was also loss in FF. On comparison of the results between the two test batches, larger amount of degradation in $\mathrm{P}_{\max }$ was observed for batch-I modules as compared to batch-II modules, as shown in Figure 3. To establish the difference between these two test batches, the change in $\mathrm{P}_{\max }$ for $\mathrm{DH}$ that was performed last in the combined test series can be observed. Here, for the two batches of DH the degradation in $\mathrm{P}_{\max }$ was $2.8 \%$ and $1.7 \%$ for batch I and II respectively. This shows that some extra effect of the preceeding test HF and TC was observed on the DH test modules, which was last in the sequence. This can be termed as lingering effect of the degradation due to preceeding tests on the subsequent one. This highlights the severity of the two tests, HF and $\mathrm{TC}$ over the $\mathrm{DH}$ test. It can also be inferred from the above results that the degradation after cyclic tests was more than the non-cyclic test $(\mathrm{DH})$ in a fixed duration of time. In order to validate the effect of TC, an additional 10 cycles of HF were performed on the batch-II HF test modules. Here, the degradation in $\mathrm{P}_{\max }$ was $5 \%$, whereas, the difference in $\mathrm{P}_{\max }$ from batch-I TC modules was 7\%. This shows the severity of the individual TC test over the HF test.

Table I Loss in electrical parameters after the test of batch I and batch II module

\begin{tabular}{lllllll}
\hline $\begin{array}{l}\text { Degradation in } \\
\text { parameters (\%) }\end{array}$ & Batch-I & \multicolumn{5}{c}{ Batch -II } \\
\hline & $\mathrm{HF}$ & $\mathrm{TC}$ & $\mathrm{DH}$ & $\mathrm{HF}$ & $\mathrm{TC}$ & $\mathrm{DH}$ \\
$\mathrm{P}_{\max }$ & 4.3 & 7.1 & 2.8 & 4.6 & 6.7 & 1.7 \\
$\mathrm{FF}$ & 3.9 & 7 & 2.2 & 4.5 & 6.5 & 1.3 \\
$\mathrm{R}_{\mathrm{s}}$ & 16.3 & 20.5 & 5 & 16 & 20.1 & 5.1 \\
\hline
\end{tabular}

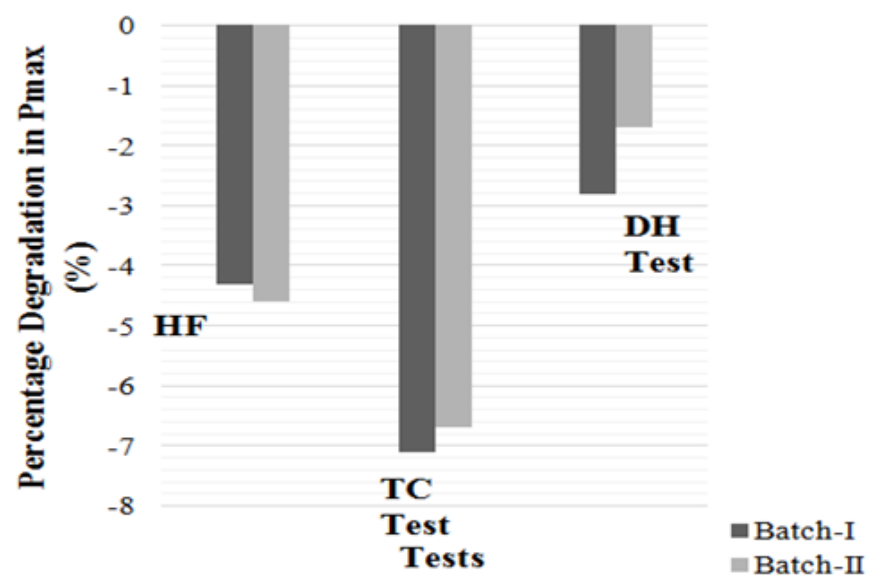

Figure 3 Comparison of power degradation after tests from batch-I and batch-II modules.

\section{Spatial characterization of the degraded modules}

EL and DLIT imaging were carried out to extract the spatially resolved information about the defects in the modules and also for establishment of correspondence with the results obtained after the $\mathrm{I}-\mathrm{V}$ analysis. In the EL images of both the batches, distinct rectangular shaped areas were observed after both HF and TC tests as shown in Figure $4 \mathrm{a}(\mathrm{ii}), 4 \mathrm{a}$ (iii) \& 5a(ii). These can be identified as broken front grid fingers. On comparison of these images with the I-V results, it can be inferred that these broken fingers have caused the increment in $\mathrm{R}_{\mathrm{s}}$. Whereas, such distinct dark areas did not appear after the DH tests for the modules as visible in Figure 4a(iv) \& 6a(ii). The increment in $\mathrm{R}$ in this case can be due to grid corrosion after the penetration of moisture through the backsheet of the module. On observing the progression of the finger defects, after TC test in the batch-I modules, the number of finger defects were observed to increase in some cell portions that appeared completely dark as shown in Figure 4a(iii). The completely dark areas can be attributed to the absolute detachment of fingers from the wafer. These regions will not participate in the current carrying process, and will cause the degradation in $\mathrm{P}_{\max }$. It was also observed that the trend of number of finger defects corresponds to the increase in $\mathrm{R}_{\mathrm{s}}$ after all the tests. On observing the EL images after DH test, no additional finger breakages were observed after the test that was conducted in succession to TC (Figure $4 \mathrm{a}(\mathrm{iv})$ ). The cycle effect in TC and HF tests caused an earlier attainment of degradation. The cyclic nature of the tests cause more fatigue stress due to alternating low and high temperature within a fixed interval. Whereas, the mode of degradation in non-cyclic DH test is due to penetration of moisture through the back sheet, which is a long-term process. Some bright spots were observed in the DLIT images after the $50 \mathrm{TC}$ cycles as seen in Figure 5b(ii). Similar spots are visible in Figure $4 \mathrm{~b}$ (iii) for the combined test batch modules after TC test. A corresponding decrease in the value of $R_{s h}$ was observed from the $I-V$ analysis, these could possibly be due to presence of localized shunts. The interpretations from both the spatial and non-spatial characterization techniques led to similar findings and were found to be in correspondance to eachother. 
(a)

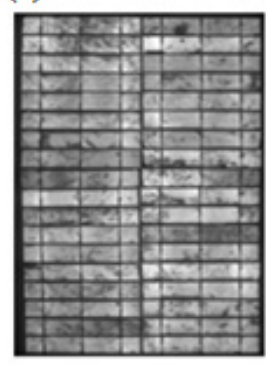

(i)

(b)

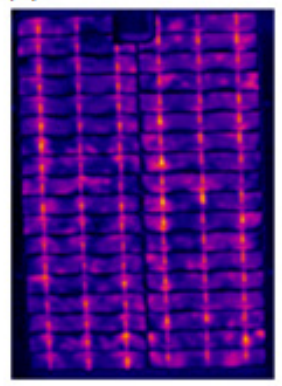

(i)

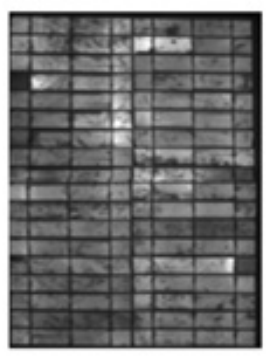

(ii)

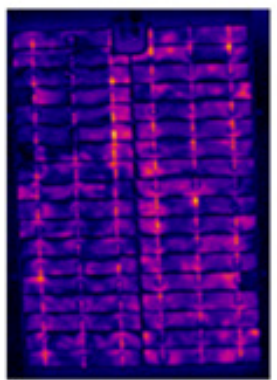

(ii)

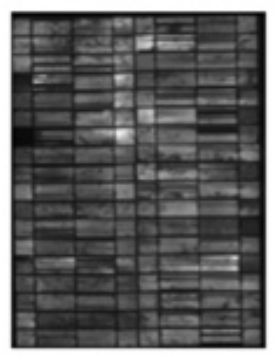

(iii)

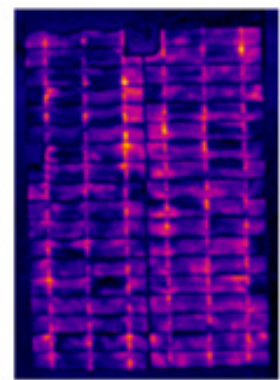

(iii)

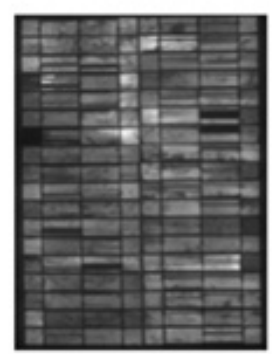

(iv)

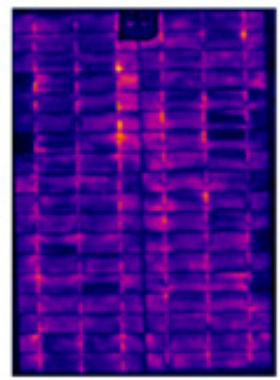

(iv)

Figure 4

(a) EL images of batch I modules (i) initially (ii) after 10 cycles of HF (iii) after 50 cycles of TC (iv) after 250h of DH.

(b) DLIT images of batch I modules (i) initially (ii) after 10 cycles of HF (iii) after 50 cycles of TC (iv) after 250hrs of DH.

(a)

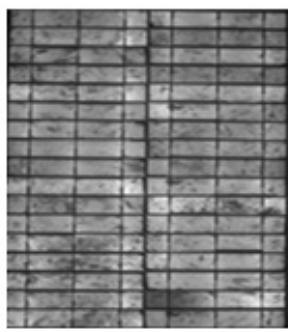

(i)

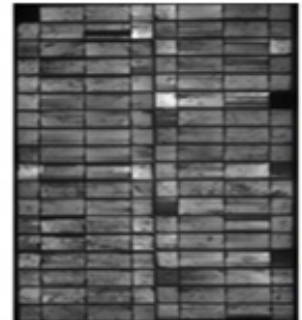

(ii)

(b)

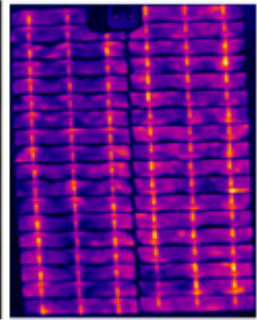

(i)

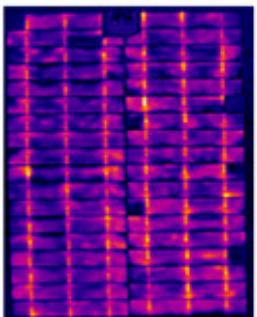

(ii)

Figure 5

(a) EL images of batch II modules (i) initially (ii) after 50 cycles of TC.

(b) DLIT images of batch II modules (i) initially (ii) after 50 cycles of TC.

(a)

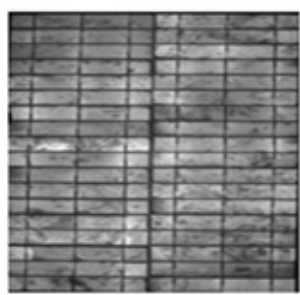

(i)

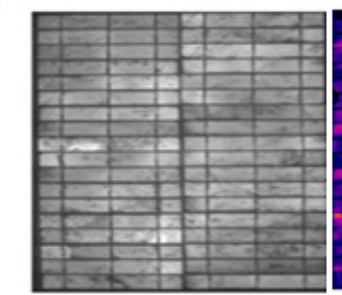

(ii)

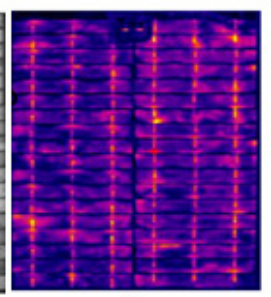

(i) (b)

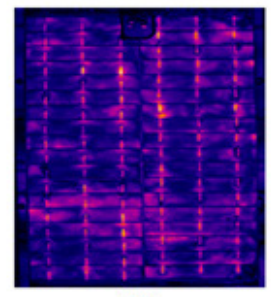

(ii)

\section{Figure 6}

(a) EL images of batch II modules (i) initially (ii) after $250 \mathrm{~h}$ of $\mathrm{DH}$.

(b) DLIT images of batch II modules (i) initially (ii) after $250 \mathrm{~h}$ of $\mathrm{DH}$. 


\section{Conclusions}

In this paper, qualitative estimation and analysis of degradation after standard tests viz; thermal cycling, humidity freeze and damp heat test has been presented by dark lock-in-thermography and electroluminescence imaging on multi-crystalline silicon PV modules. Illuminated I-V characteristic parameters were used in support of the spatial images for quantification of degradation in terms of electrical parameters after the standard tests. Results obtained from the analysis have been used for comparison of different amount of degradation observed after the tests. From the investigation, cyclic tests were found to cause more degradation in comparion to the non-cycling test. Finger defects were found to be the major cause of degradation after the cylic tests, visible as dark rectangular regions in the EL images. Corresponding loss in power was observed from the I-V analysis dominantly due to loss in fill factor attributed to increment in series resistance. TC test was found to be the most severe causing maximum degradation in comparison to DH test in a fixed duration of time. A lingering effect of TC and HF test was observed on DH test which showed the dependence of the tests in a sequence. The study was able to comment on the severity of individual tests and sequences along with identification of the common modes of degradation by exploiting the available characterization techniques.

\section{Acknowledgments}

Funding was provided by Department of Science and Technology (DST) in India and Research Councils UK (RCUK) Energy Programme in UK.

\section{Conflicts of interest}

The author declares that there is no conflict of interest.

\section{References}

1. IEC 61215. Crystalline Silicon Terrestrial Photovoltaic (PV) modulesDesign qualification and type approval, ( $\left.2^{\text {nd }} e d n\right) .2005$.
2. Dumas LN, Shumka A. Photovoltaic Module Reliability Improvement through Application Testing and Failure Analysis. IEEE Trans Reliab.1982;3(1):228-234.

3. Ferrara C, Philipp D. Why do PV modules fail? Energy Procedia, International conference on materials for advanced technologies, Suntech. Singapore pp. 2012;379-387.

4. Khatri R, Agarwal S, Saha I, et al. Study on long term reliability of photovoltaic modules and analysis of power degradation using accelerated aging tests and electroluminescence technique. Energy Procedia. 2011;8(1):396-401

5. Fuyuki T, Kondo H, Kaji $\mathrm{Y}$, et al. Analytic findings in the electroluminescence characterization of crystalline silicon solar cells. $J$ Appl Phys. 2007;101(2):1-1.

6. Fuyuki T, Kitiyanan A. Photographic diagnosis of crystalline silicon solar cells utilizing electroluminescence. Appl Phys A Mater Sci Process. 2009;96(1):189-196.

7. Roy S, Somasundaran P, Gupta R. Estimation of shunt resistance by electroluminescence imaging. 29th European Photovoltaic Solar Energy Conference and Exhibition. 2014.

8. Chaturvedi P, Hoex B, Walsh TM. Broken metal fingers in silicon wafer solar cells and PV modules. Sol Energy Mater Sol Cells. 2013;108(1):7881 .

9. Breitenstein O, Warta W, Langenkamp M. Lock-In Thermography-basics and use for evaluating electronic devices and materials. ( $2^{\text {nd }}$ edn). 2010.

10. Gupta R, Somasundaran P, Nandi DK. Electrical simulation and characterization of shunts in solar cells. Appl Mech Mater. 2011;110 116:2453-2457.

11. Breitenstein O, Langenkamp M, Lang O, Schirrmacher A. Shunts due to laser scribing of solar cells evaluated by highly sensitive lock-in thermography. Sol Energy Mater Sol Cells. 2001;65(1-4):55-62.

12. Somasundaran P, Sinha A, Gupta R. Simulation and characterization of spatial variation of shunts in industrial solar cells by PSPICE and Dark Lock-in Thermography. 27th European Photovoltaic Solar Energy Conference and Exhibition. 2012. 